\title{
Improved Access to the Bone Marrow Space by Multiple Perforations of the Bundle Bone after Tooth Extraction - A Technical Note
}

\section{Azadeh Esfandeyari ', Georg D. Strbac ', Andreas Stavropoulos ${ }^{2}$, Kristina Bertl ${ }^{2}$, Christian UIm}

\author{
Division of Oral Surgery, University Clinic of Dentistry, Medical University of Vienna, Austria \\ Divison of Periodontology, Faculty of Odontology, University of Malmö, Sweden
}

\begin{abstract}
Background
The dental alveolus is lined by a thin cortical layer ("bundle bone", "alveolar bone proper", "cribriform plate", "lamina dura"), that can impede access to the bone marrow and its vasculature. During unassisted socket healing, the bundle bone is gradually resorbed allowing tissue resources from the bone marrow to enter into the socket space. An optimized wound healing process, either during unassisted socket healing or during ridge preservation procedures, with autogenous bone and/or any bone/collagen substitute material, depends at least partly on an adequate vascularization of the socket space. This ensures sufficient recruitment of osteoblast and osteoclast precursor cells and facilitates fast bone regeneration and/or uneventful integration of the augmentation material.

During guided bone regeneration (GBR) and block augmentation procedures, several small perforations of the cortical bone at the recipient site are recommended to open the bone marrow space, increase the blood inflow, and allow migration of osteogenic tissue resources into the defect area. Hence, the cortical character of the alveolar bone proper of an empty dental alveolus may be seen in a similar manner as the cortical layer at the recipient site during GBR; it would thus be reasonable to suggest that perforations of the palatal/ lingual wall after tooth extraction might result in an improved unassisted socket healing and corticalization of the entrance [I, 2] as well as in an enhanced bone formation and/or incorporation of any grafting material, finally resulting in a reduced volume reduction of the alveolar ridge (i.e., improved ridge preservation) [3].
\end{abstract}

\section{Hypothesis}

The present technical note describes an easy treatment step after tooth extraction aiming to improve socket healing with or without any ridge preservation procedure, by facilitating an increased blood inflow into the dental alveolus.

\section{Materials and Methods}

The additional treatment step is performed after regular tooth extraction independent of whether unassisted socket healing or any ridge preservation procedure is planned. Depending on the size of the extraction socket, about 6 to 10 small perforations of the palatal/ lingual and - in the absence of neighboring teeth - of the mesial/distal aspect of the bundle bone are performed in relatively close proximity to each other (Fig.I)

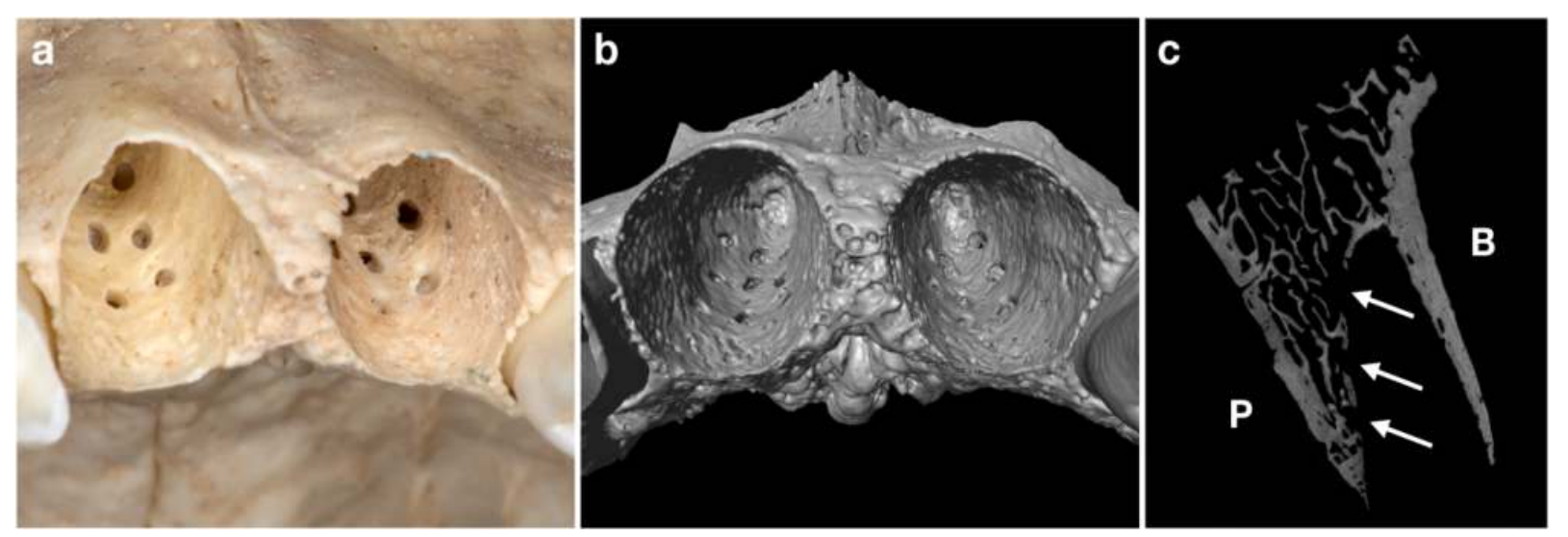

Fig. I. a) Human maxillary cadaveric specimen showing 2 empty central incisal alveoli after performing multiple perforations of the palatal bundle bone in relatively close proximity to each other (i.e., 2 to $3 \mathrm{~mm}$ distance inbetween); b) occlusal view of a micro computed-tomography scan of the same specimen displaying about 10 small perforations in each socket; c) cross-sectional slice of the same scan illustrating 3 palatal perforations of the alveolar bone proper extending into the trabecular bone (white arrows). P, palatal; B, buccal.

\section{References}

ITrombelli, L., Farina, R., Marzola, A., Bozzi, L., Liljenberg, B., \& Lindhe, J. Modeling and remodeling of human extraction sockets. Clinical Periodontol $35,630,2008$

2 Bertl K, Kukla EB, Albugami B, Beck F, Gahleitner A, Stavropoulos A.Timeframe of socket cortication after tooth extraction:A retrospective radiographic study. Clin Oral Implants Res 29:130, 2018

3 Mardas N, Trullenque-Eriksson A, MacBeth N, Petrie A, Donos N. Does ridge preservation following tooth extraction 26:180, impl5

4 Sicher H, DuBrul E (eds). Oral Anatomy. $5^{\text {th }}$ Ed., Saint Louis, MO, CV Mosby Company, 1975, pp 4I3 - 429

5 Cha JK, Kim CS, Choi SH, Cho KS, Chai JK, Jung UW. The influence of perforating the autogenous block bone and the recipient bed in dogs. Part II: histologic analysis. Clin Oral Implants Res 23:987, 2012

6 Schmid J, Wallkamm B, Hämmerle CH, Gogolewski S, Lang NP:The significance of angiogenesis in guided bone regeneration. A case report of a rabbit experiment. Clin Oral Implants Res 8:244, 1997

7 Lee JS, Shin HK, Yun JH, Cho KS: Randomized Clinical Trial of Maxillary Sinus Grafting using Deproteinized Porcine and Bovine Bone Mineral. Clin Implant Dent Relat Res 19:140, 2017

The perforations are performed relatively fast and easy with a small round bur with a diameter $<1 \mathrm{~mm}$ (Fig. 2).

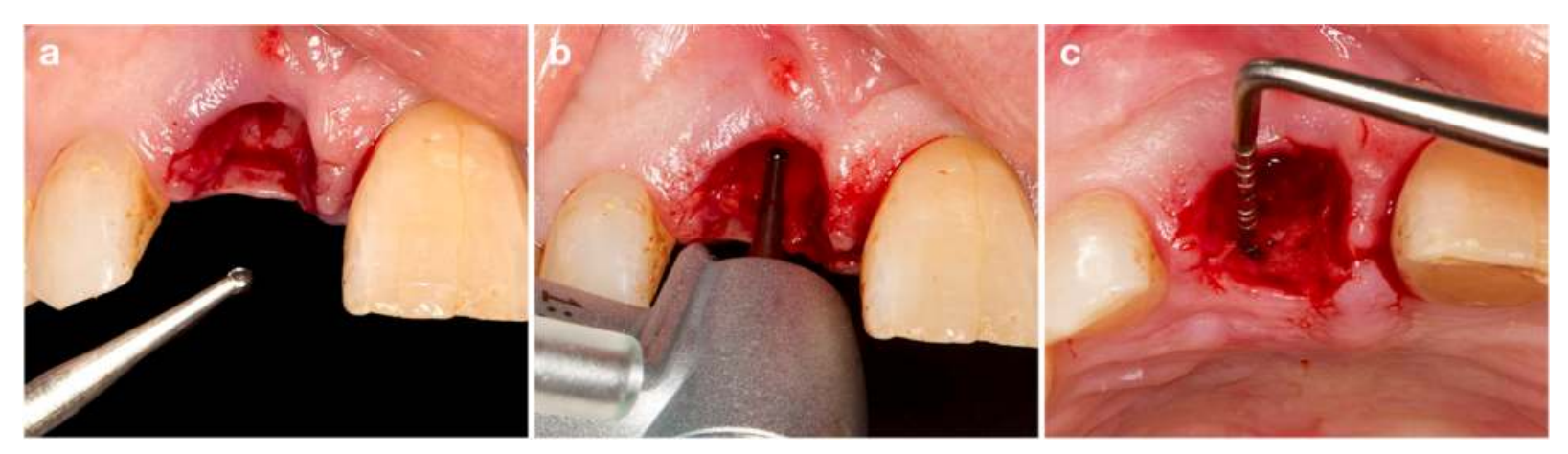

Fig. 2. a and b) Clinical case treated by the proposed technique. After tooth extraction, the palatal alveolar bone proper is perforated several times with a small round bur; c) the perforations should extend into the trabecular
bone, as illustrated by a periodontal probe entering one of the perforations.

The perforations should extend into the trabecular bone to provoke bleeding, but care should be taken not to drill too deep in order to maintain the integrity of the external plate of the alveolar process at the palatal/lingual aspect and to preserve any neighboring structures. On the buccal side, especially on the anterior regions of the jaws, the bundle bone is most often fused with the external plate of the alveolar process [4], and thus this aspect of the socket is mainly composed of cortical- and only minor trabecular bone, explaining the increased resorption observed from this aspect; thus the buccal bundle bone should be spared, and no perforations should be made. After all perforations are performed any further treatment steps for ridge preservation may be continued (i.e., hard and/or soft tissue grafting, wound closure, etc.).

\section{Results}

Up to now, several patients have been treated using this modified ridge preservation technique at the Division of Oral Surgery (University Clinic of Dentistry, Medical University of Vienna, Austria). No complications have been observed in terms of the access to perform the perforations neither in terms of any conceivable post-surgical events related to the modification (e.g., excessive hematoma, wound dehiscences, etc.); further, the procedure was well accepted by the patients.

Naturally, the lack of standardized/controlled assessment of this technique does not allow - up to now - any conclusion on the hypothesis that perforations of the bundle bone result in any tangible clinical or histological benefit; e.g., reduced rate of dry sockets, enhanced bone formation and corticalization, and/or graft incorporation resulting in reduced alveolar ridge resorption, etc.. Preclinical trials aiming for micro computed-tomography and histological outcome assessment have been launched to assess the effect size of this simple modification during alveolar ridge preservation procedures.

\section{Conclusion}

The above-described additional surgical step after regular tooth extraction is based on the rationale that one major factor during bone healing and incorporation of a grafting material is the sufficient vascularization, which provides the necessary cells and factors $[5,6,7]$; disturbance or lack of vascularization may result in delayed healing and/ or compromised integration of the grafting material) [8]. Preclinical and clinical trials have provided histological evidence of the relevance of sufficient capillary ingrowth for new bone formation and, consequently, of the positive effect of such perforations during GBR and block augmentation procedures; i.e., faster incorporation and/or reduced resorption of bone grafts, and larger amounts of bone gain, after perforation of the cortical layer of the recipient site compared to cases without perforations has been observed [9]. 